\title{
Asymmetric Allylic Alkylation of Ketone Enolates: An Asymmetric Claisen Surrogate.
}

\author{
Erin C. Burger and Jon A. Tunge* \\ Department of Chemistry, University of Kansas, Lawrence, KS 66045

\section{Supporting Information}

\section{Experimental}

Materials. Benzene was dried over sodium metal. All other materials were used as received. $\beta$-ketoesters $\mathbf{1 a}-\mathbf{1 h}$ were prepared by the addition of diketene to the corresponding allylic alcohols. ${ }^{1} \quad \beta$-ketoester 1f was prepared by the DMAP catalyzed condensation of $\beta$-ketoethylester and allylic alcohol. ${ }^{2} \beta$-ketoesters 1i-1l were prepared by the condensation of the corresponding acid chloride with Meldrum's acid ${ }^{3}$ followed by addition of the appropriate allylic alcohol. ${ }^{4} \mathrm{H}$ NMR spectra were obtained on a Bruker Avance 400 or a Bruker Avance 500 DRX spectrometer and referenced to residual protio solvent signals. Structural assignments are based on ${ }^{1} \mathrm{H},{ }^{13} \mathrm{C}$, DEPT-135, COSY, and HMQC spectroscopies.

General procedure for catalytic rearrangements: In a Schlenk tube under Ar, allyl$\beta$-ketoester $(1.2 \mathrm{mmol})$ and either $\mathrm{Pd}\left(\mathrm{PPh}_{3}\right)_{4}(10 \mathrm{~mol} \%)$ or the combination of $\mathrm{Pd}_{2}(\mathrm{dba})_{3}$ (5 mol \%) and Trost ligand (10 mol \%) were dissolved in $4 \mathrm{~mL}$ of benzene. The reaction was allowed to stir under Ar for the reported time. Following solvent evaporation the crude product was purified via flash chromatography $\left(\mathrm{SiO}_{2}, 10 \% \mathrm{Et}_{2} \mathrm{O}: \mathrm{Hex}\right)$.

\footnotetext{
${ }^{1}$ Collado, I.; Pedregal, C.; Mazon, A.; Espinosa, J. F.; Blanco-Urgoiti, J.; Schoepp, D. D.; Wright, R. A.; Johnson, B. G.; Kingston, A. E. J. Med.Chem. 2002, 45, 3619-3629.

${ }^{2}$ Gilbert, J. C.; Kelly, T. A. J. Org. Chem. 1998, 53, 449-450.

${ }^{3}$ Svenstrup, N.; Simonsen, K.; Thorup, N.; Brodersen, J.; Dehaen, W.; Becher, J. J. Org. Chem. 1999, 64, 2814-2820.

${ }^{4}$ Yuste, F.; Brena, F.; Barrios, H.; Sanchez-Obregon, R.; Ortiz, B.; Walls, F. Synth. Commun. 1988, 18, 735-739.
} 


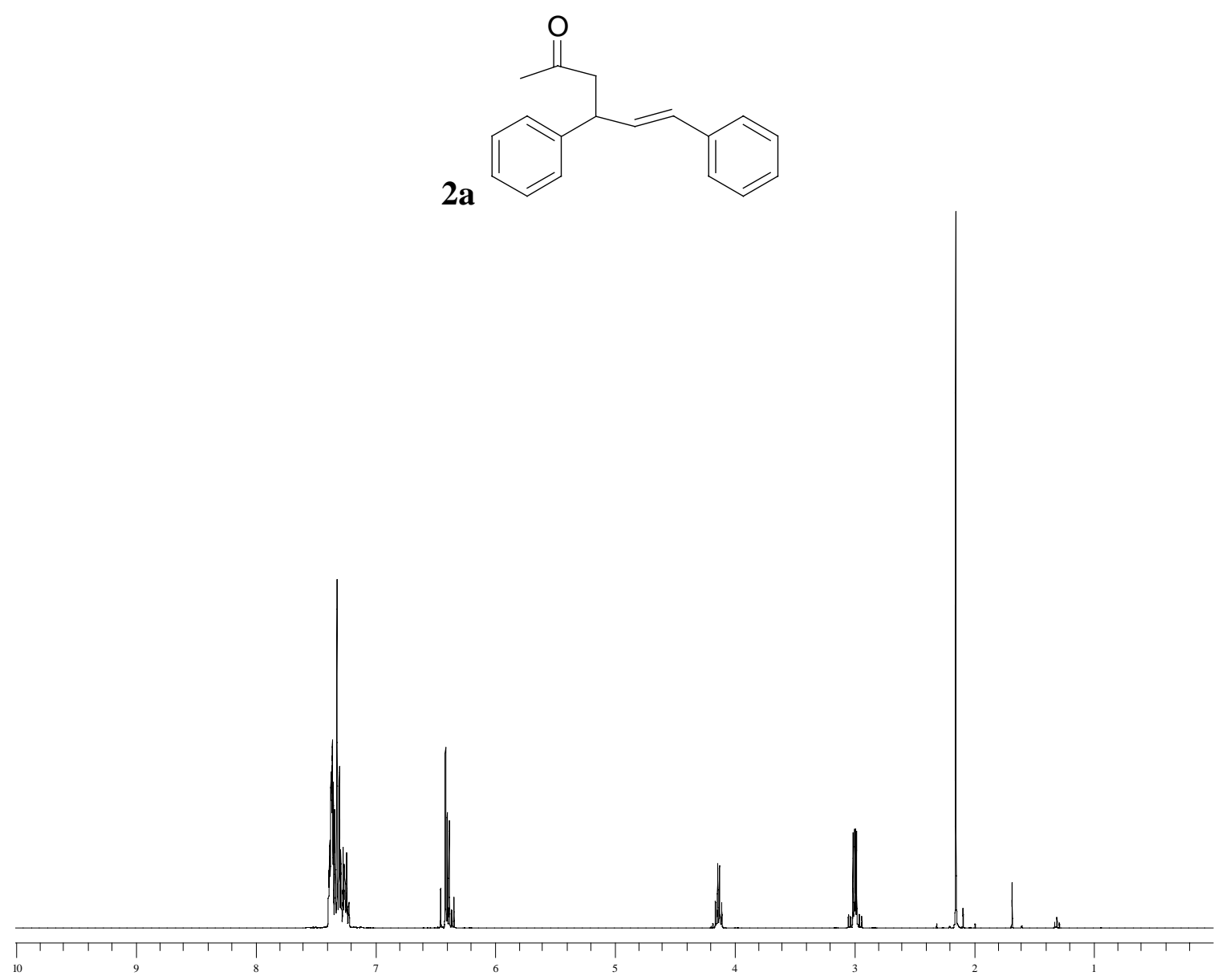




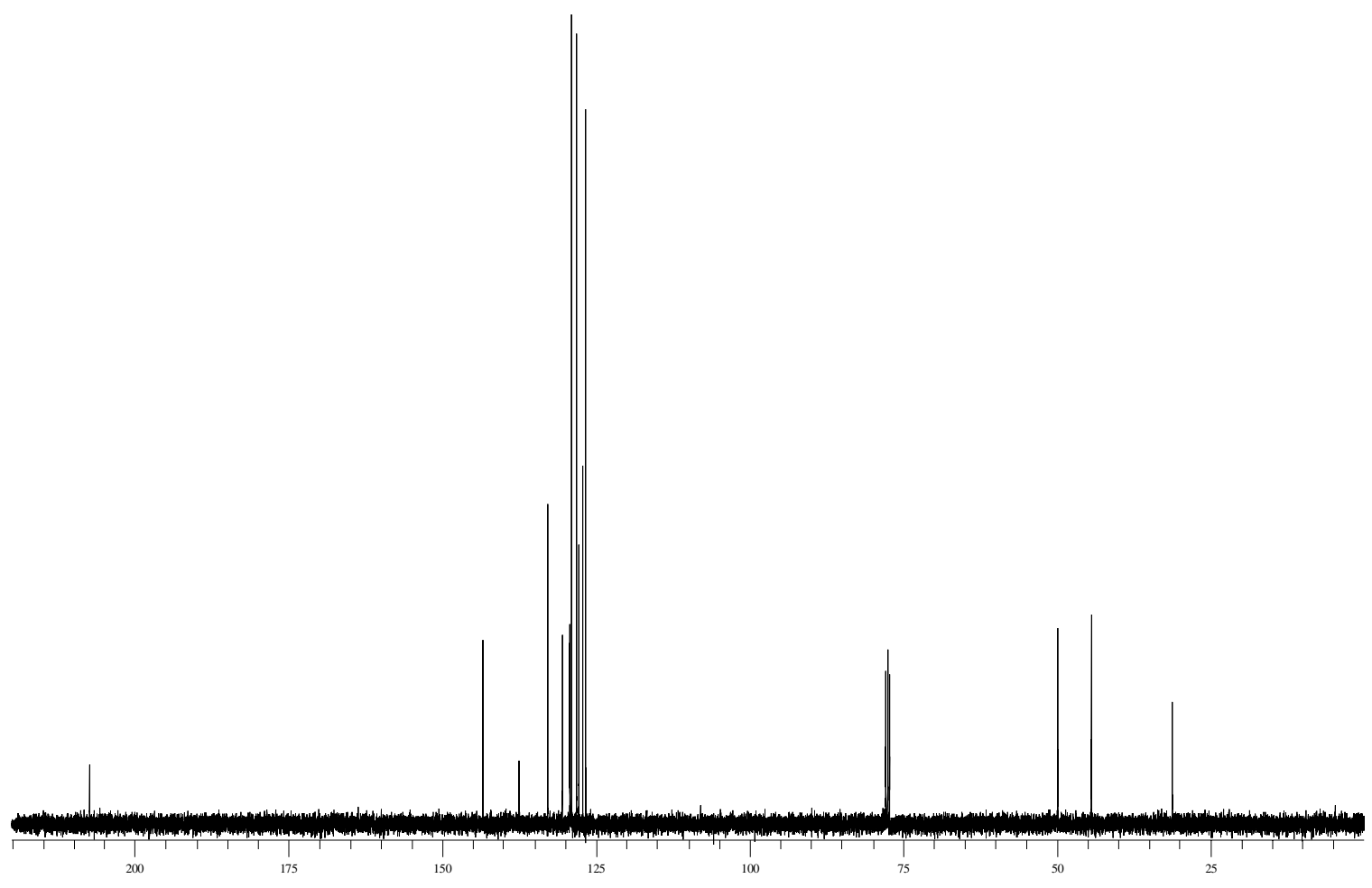

2a ${ }^{5}$ yellow oil: ${ }^{1} \mathrm{H}$ NMR $\left(400 \mathrm{MHz}, \mathrm{CDCl}_{3}\right) \delta 7.30(\mathrm{~m}, 10 \mathrm{H}$ : arom $\mathrm{H}), 6.38(\mathrm{~m}, 2 \mathrm{H}$ : ambiguous $\mathrm{CH}=$ ), 4.12 (app. q, $J=7 \mathrm{~Hz}, 1 \mathrm{H}: \mathrm{CH}$ ), 3.01 (dd, $J=7 \mathrm{~Hz}, 16 \mathrm{~Hz}, 1 \mathrm{H}$ : diastereotopic $\left.\mathrm{CH}_{2}\right) 2.95\left(\mathrm{dd}, J=7 \mathrm{~Hz}, 16 \mathrm{~Hz}, 1 \mathrm{H}\right.$ : diastereotopic $\left.\mathrm{CH}_{2}\right), 2.14$ (s, 3H: $\left.\mathrm{CH}_{3}\right) .{ }^{13} \mathrm{C} \mathrm{NMR}\left(75 \mathrm{MHz}, \mathrm{CDCl}_{3}\right) \delta 207.36(\mathrm{C}=\mathrm{O}), 143.35$ (arom. C), 137.49 (arom. C), $132.76(\mathrm{CH}=), 130.39(\mathrm{CH}=), 129.13$ (arom. $\mathrm{CH}), 128.91$ (arom. $\mathrm{CH}), 128.06$ (arom. $\mathrm{CH}$ ), 127.75 (arom. CH), 127.12 (arom. CH), 126.65 (arom. $\mathrm{CH}$ ), $49.82\left(\mathrm{CH}_{2}\right), 44.36$ $(\mathrm{CH}), 31.20\left(\mathrm{CH}_{3}\right)$. IR $\left(\mathrm{CH}_{2} \mathrm{Cl}_{2}\right): v_{\max } 1710,1490,1255$. HRMS calcd for $\mathrm{C}_{18} \mathrm{H}_{18} \mathrm{O}[\mathrm{M}+]$ 250.1358 , found 250.1352 .

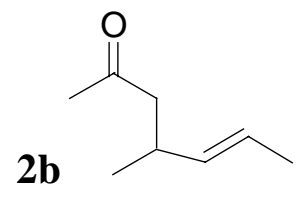

\footnotetext{
${ }^{5}$ Oi, S.; Honma, Y.; Inoue, Y. Org. Lett. 2002, 4, 667.
} 

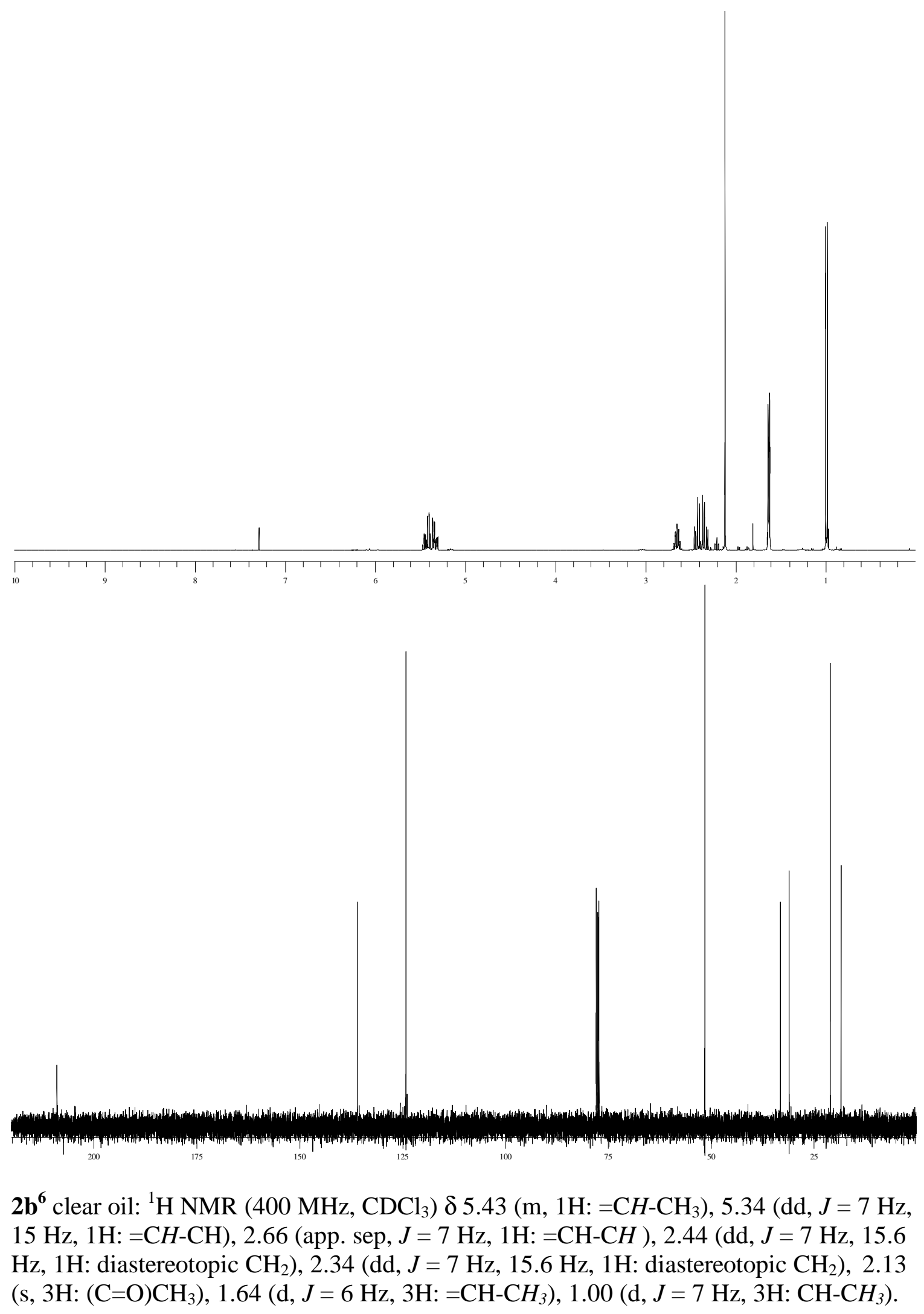

${ }^{6}$ Tsuji, J.; Yamada, T.; Minami, I.; Yuhara, M.; Nisar, M.; Shimizu, I. J. Org. Chem. 1987, 52, 2988-95. 
${ }^{13} \mathrm{C}$ NMR $\left(75 \mathrm{MHz}, \mathrm{CDCl}_{3}\right) \delta 208.92(\mathrm{C}=\mathrm{O}), 135.90(=\mathrm{CH}-\mathrm{CH}), 124.14\left(=\mathrm{CH}-\mathrm{CH}_{3}\right)$, $51.40\left(\mathrm{CH}_{2}\right), 33.11(=\mathrm{CH}-\mathrm{CH}), 30.90\left((\mathrm{C}=\mathrm{O}) \mathrm{CH}_{3}\right), 20.93\left(\mathrm{CH}-\mathrm{CH}_{3}\right), 18.28\left(=\mathrm{CH}-\mathrm{CH}_{3}\right)$. IR $\left(\mathrm{CDCl}_{3}\right): v_{\max } 1705,1454,1357$. HRMS calcd for $\mathrm{C}_{8} \mathrm{H}_{15} \mathrm{O}[\mathrm{M}+\mathrm{H}] 127.1123$, found 127.1105. GC (Chiraldex B-TA : Hold $50^{\circ} \mathrm{C}$ for $5 \mathrm{~min}$., ramp $1^{\circ} \mathrm{C} / \mathrm{min}$ to $75^{\circ} \mathrm{C}$ ) $\mathrm{t}_{\mathrm{r}}=$ 27.6, $29.3 \mathrm{~min}$.

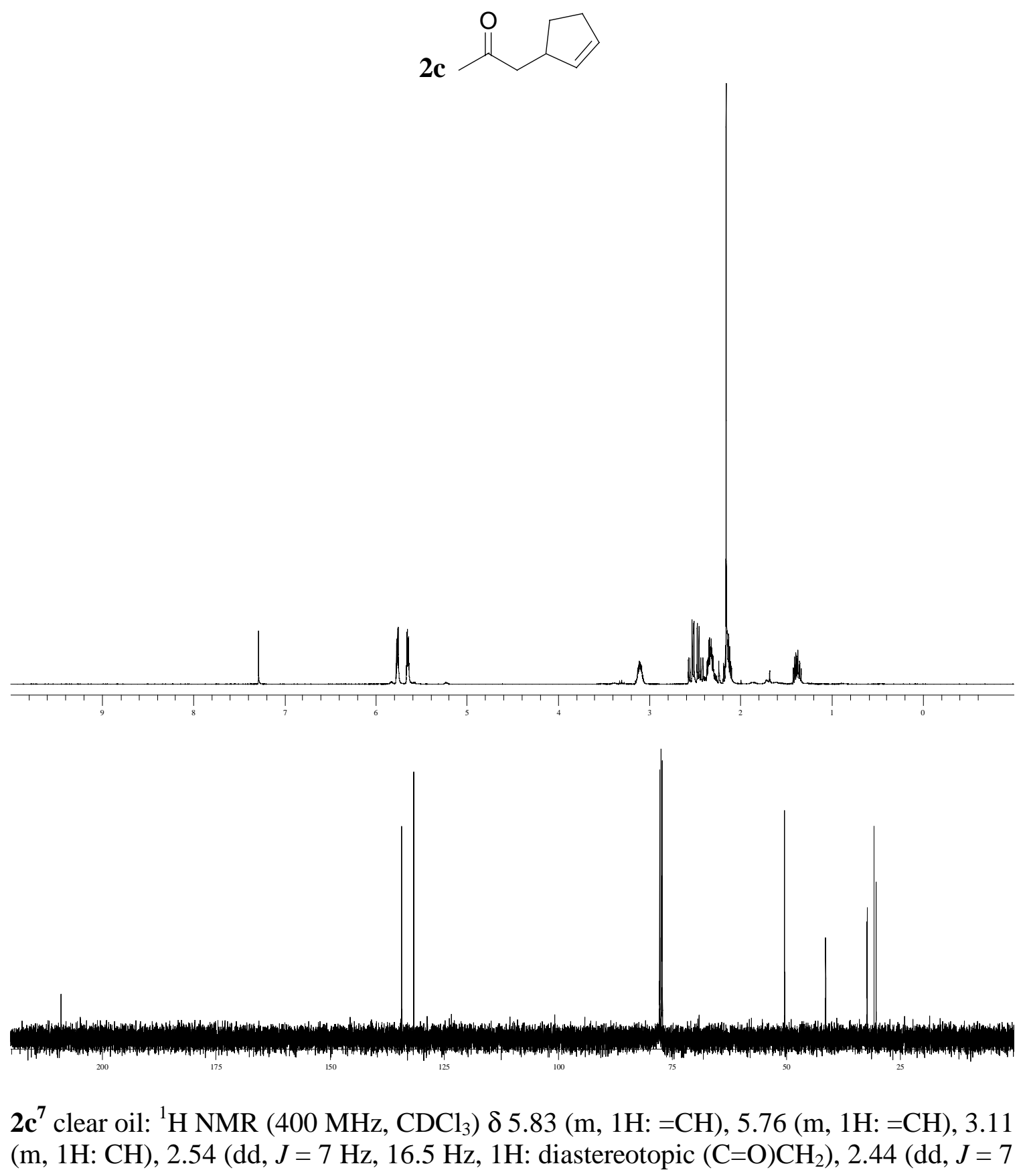

${ }^{7}$ Hassner, A. Naumann, F. Chem. Ber. 1988, 121, 1823-6. 
$\mathrm{Hz}, 16.5 \mathrm{~Hz}, 1 \mathrm{H}$ : diastereotopic $\left.(\mathrm{C}=\mathrm{O}) \mathrm{CH}_{2}\right), 2.32\left(\mathrm{~m}, 2 \mathrm{H}\right.$ : ambiguous $\left.\mathrm{CH}_{2}\right), 2.16(\mathrm{~s}, 3 \mathrm{H}$ : $\left.\mathrm{CH}_{3}\right), 2.14\left(\mathrm{~m}, 1 \mathrm{H}\right.$ : ambiguous diastereotopic $\left.\mathrm{CH}_{2}\right), 1.38$ (m, $1 \mathrm{H}$ : ambiguous

diastereotopic $\left.\mathrm{CH}_{2}\right) .{ }^{13} \mathrm{C}$ NMR $\left(75 \mathrm{MHz}, \mathrm{CDCl}_{3}\right) \delta 209.01(\mathrm{C}=\mathrm{O}), 134.30(5.76)(=\mathrm{CH})$, $131.67(5.83)(=\mathrm{CH}), 50.32\left((\mathrm{C}=\mathrm{O}) \mathrm{CH}_{2}\right), 41.36(\mathrm{CH}), 32.21$ (ambiguous $\left.\mathrm{CH}_{2}\right), 30.70$ $\left(\mathrm{CH}_{3}\right), 30.20$ (ambiguous $\left.\mathrm{CH}_{2}\right)$. IR $\left(\mathrm{CDCl}_{3}\right)$ : $v_{\max } 1700,1403,1362$. HRMS calcd for $\mathrm{C}_{8} \mathrm{H}_{12} \mathrm{O}[\mathrm{M}+\mathrm{H}]$ 125.0966, found 125.0970. GC (Chiraldex B-TA : Hold $50^{\circ} \mathrm{C}$ for 5 min., ramp $0.5^{\circ} \mathrm{C} / \mathrm{min}$ to $75^{\circ} \mathrm{C}$ ) $\mathrm{t}_{\mathrm{r}}=47.3,48.5 \mathrm{~min}$.

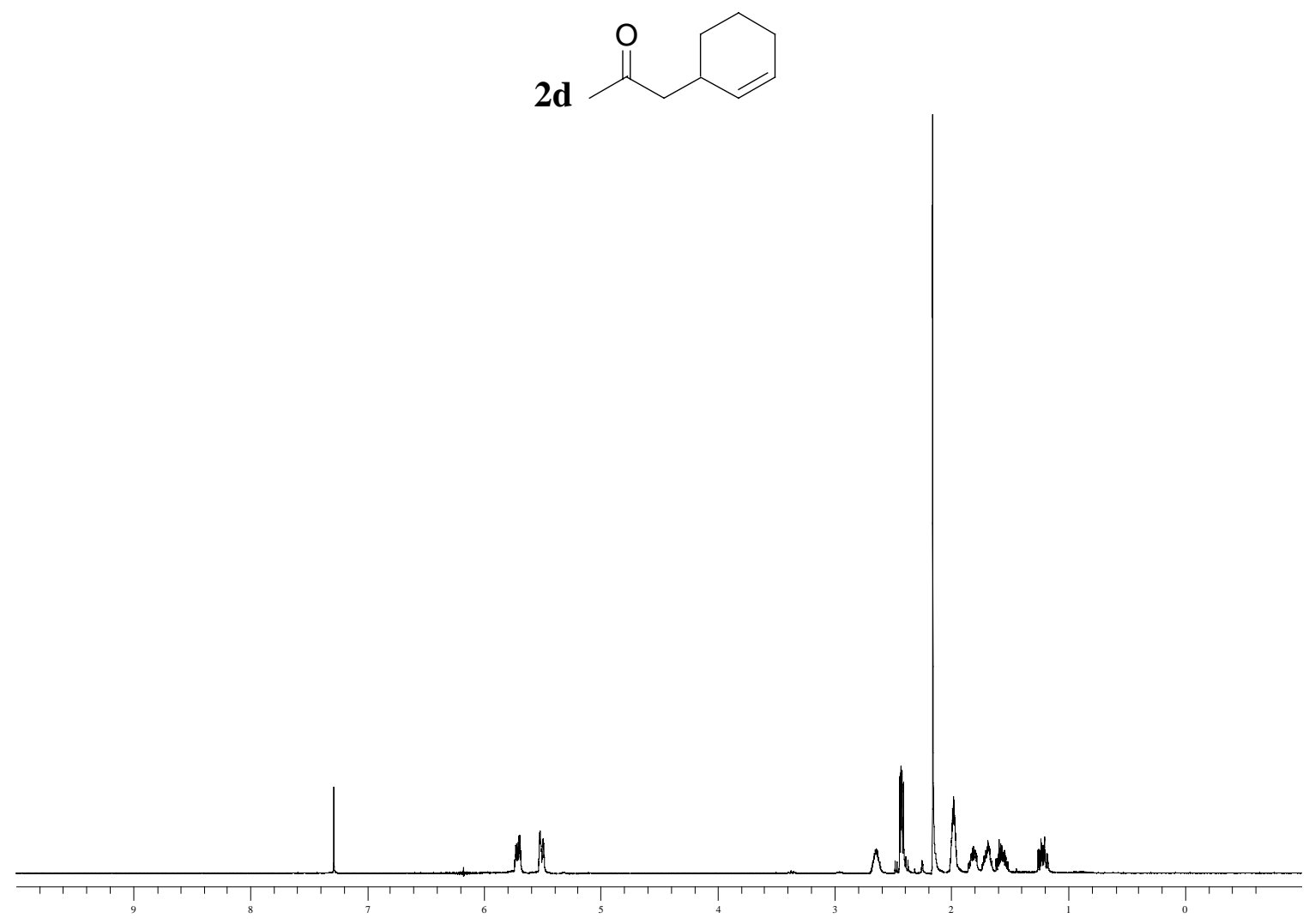




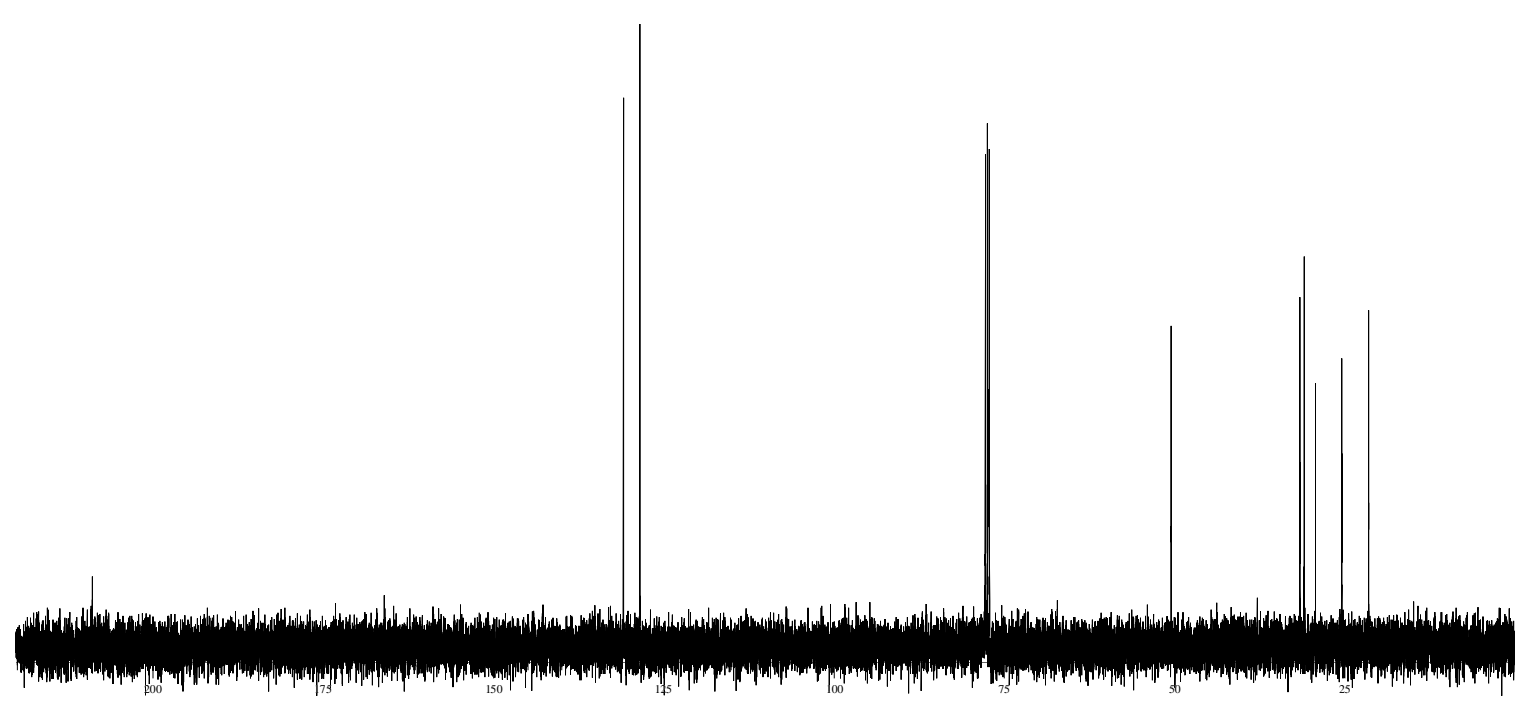

$\mathbf{2 d}^{8}$ clear oil: ${ }^{1} \mathrm{H}$ NMR $\left(400 \mathrm{MHz}, \mathrm{CDCl}_{3}\right) \delta 5.71(\mathrm{~m}, 1 \mathrm{H}:=\mathrm{CH}), 5.51(\mathrm{~m}, 1 \mathrm{H}:=\mathrm{CH}), 2.65$ $(\mathrm{m}, 1 \mathrm{H}:=\mathrm{CH}-\mathrm{CH}), 2.46\left(\mathrm{dd}, J=7 \mathrm{~Hz}, 16.3 \mathrm{~Hz}, 1 \mathrm{H}\right.$ : diastereotopic $\left.(\mathrm{C}=\mathrm{O}) \mathrm{CH}_{2}\right), 2.40(\mathrm{dd}$, $J=7 \mathrm{~Hz}, 16.3 \mathrm{~Hz}, 1 \mathrm{H}$ : diastereotopic $\left.(\mathrm{C}=\mathrm{O}) \mathrm{CH}_{2}\right), 2.16\left(\mathrm{~s}, 3 \mathrm{H}: \mathrm{CH}_{3}\right), 1.98(\mathrm{~m}, 2 \mathrm{H}$ : ambiguous $\left.\mathrm{CH}_{2}\right), 1.81\left(\mathrm{~m}, 1 \mathrm{H}\right.$ : ambiguous diastereotopic $\left.\mathrm{CH}_{2}\right), 1.69(\mathrm{~m}, 1 \mathrm{H}$ : ambiguous diastereotopic $\left.\mathrm{CH}_{2}\right), 1.56\left(\mathrm{~m}, 1 \mathrm{H}\right.$ : ambiguous diastereotopic $\left.\mathrm{CH}_{2}\right), 1.23(\mathrm{~m}, 1 \mathrm{H}$ :

ambiguous diastereotopic $\left.\mathrm{CH}_{2}\right) .{ }^{13} \mathrm{C} \mathrm{NMR}\left(75 \mathrm{MHz}, \mathrm{CDCl}_{3}\right) \delta 208.81(\mathrm{C}=\mathrm{O}), 130.85$ (5.51) $(=\mathrm{CH}), 128.38(5.71)(=\mathrm{CH}), 50.46\left((\mathrm{C}=\mathrm{O}) \mathrm{CH}_{2}\right), 31.55(\mathrm{CH}), 30.92\left(\mathrm{CH}_{3}\right), 29.29$ $(1.81,1.23)$ (ambiguous $\left.\mathrm{CH}_{2}\right), 25.43$ (1.98) (ambiguous $\left.\mathrm{CH}_{2}\right), 21.43(1.69,1.56)$ (ambiguous $\left.\mathrm{CH}_{2}\right)$. IR $\left(\mathrm{CDCl}_{3}\right): v_{\max } 1700,1450,1357$. HRMS calcd for $\mathrm{C}_{9} \mathrm{H}_{15} \mathrm{O}[\mathrm{M}+\mathrm{H}]$ 139.1123, found 139.1129. GC (Chiraldex B-TA : Hold $50^{\circ} \mathrm{C}$ for $5 \mathrm{~min}$. , ramp $0.5^{\circ} \mathrm{C} / \mathrm{min}$ to $\left.85^{\circ} \mathrm{C}\right) \mathrm{t}_{\mathrm{r}}=71.9,72.8 \mathrm{~min}$.<smiles>[Z20]C(=O)CC1C=CCCC1</smiles>

\footnotetext{
${ }^{8}$ Monti, S.; Cowherd, F. G.; McAninch, T. W. J. Org. Chem. 1975, 40, 858-62.
} 

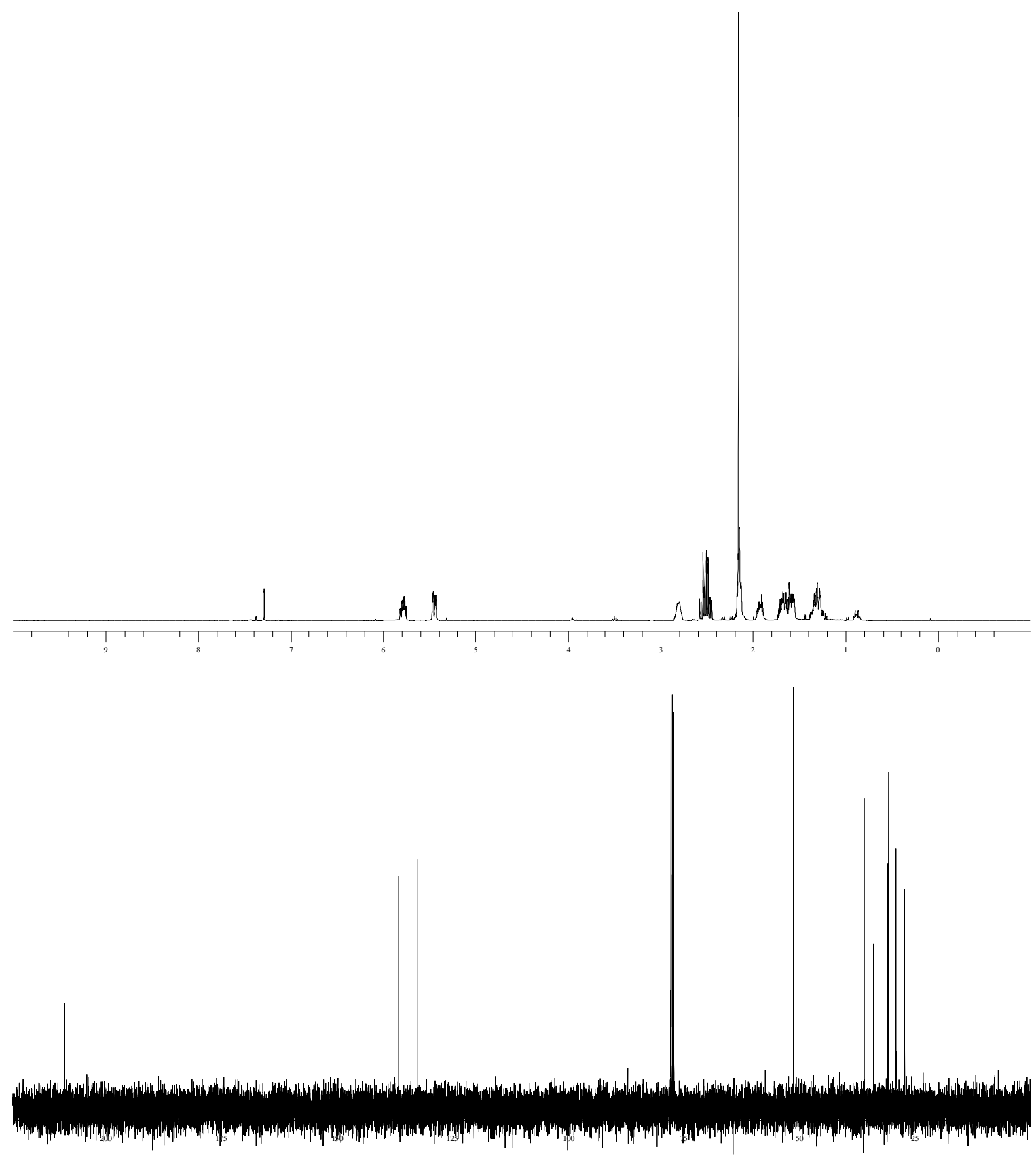

2e clear oil: ${ }^{1} \mathrm{H}$ NMR (400 MHz, $\left.\mathrm{CDCl}_{3}\right) \delta 5.78$ (m, $\left.1 \mathrm{H}:=\mathrm{CH}-\mathrm{CH}_{2}\right), 5.45(\mathrm{dd}, J=4 \mathrm{~Hz}$, $11 \mathrm{~Hz}, 1 \mathrm{H}:=\mathrm{CH}-\mathrm{CH}), 2.81(\mathrm{~m}, 1 \mathrm{H}: \mathrm{CH}), 2.55(\mathrm{dd}, J=7 \mathrm{~Hz}, 16.4 \mathrm{~Hz}, 1 \mathrm{H}$ : diastereotopic $\left.(\mathrm{C}=\mathrm{O}) \mathrm{CH}_{2}\right), 2.47\left(\mathrm{dd}, J=7 \mathrm{~Hz}, 16.4 \mathrm{~Hz}, 1 \mathrm{H}\right.$ : diastereotopic $\left.(\mathrm{C}=\mathrm{O}) \mathrm{CH}_{2}\right), 2.15(\mathrm{~s}, 3 \mathrm{H}$ :

$\mathrm{CH}_{3}$ ), 2.15 (m, 2H: ambiguous $\mathrm{CH}_{2}$ ), 1.92 (m, 1H: ambiguous diastereotopic $\mathrm{CH}_{2}$ ), 1.64 (broad m, 3H), 1.29 (m, 2H: ambiguous $\left.\mathrm{CH}_{2}\right) \cdot{ }^{13} \mathrm{C} \mathrm{NMR}\left(75 \mathrm{MHz}, \mathrm{CDCl}_{3}\right) \delta 208.86$ $(\mathrm{C}=\mathrm{O}), 136.60(=\mathrm{CH}-\mathrm{CH}), 132.43\left(=\mathrm{CH}-\mathrm{CH}_{2}\right), 51.20\left((\mathrm{C}=\mathrm{O}) \mathrm{CH}_{2}\right), 35.93(\mathrm{CH}), 33.88$ (1.59, 1.29) (ambiguous $\mathrm{CH}_{2}$ ), $30.79\left(\mathrm{CH}_{3}\right), 30.65$ (ambiguous $\left.\mathrm{CH}_{2}\right), 29.10$ (ambiguous $\mathrm{CH}_{2}$ ), 27.20 (ambiguous $\mathrm{CH}_{2}$ ). IR $\left(\mathrm{CDCl}_{3}\right): v_{\max } 1710,1450,1357$. HRMS calcd for $\mathrm{C}_{10} \mathrm{H}_{17} \mathrm{O}[\mathrm{M}+\mathrm{H}]$ 153.1279, found 153.1286. GC (Chiraldex B-TA : Hold 80 $\left.{ }^{\circ} \mathrm{C}\right) \mathrm{t}_{\mathrm{r}}=76.2$, $77.1 \mathrm{~min}$. 


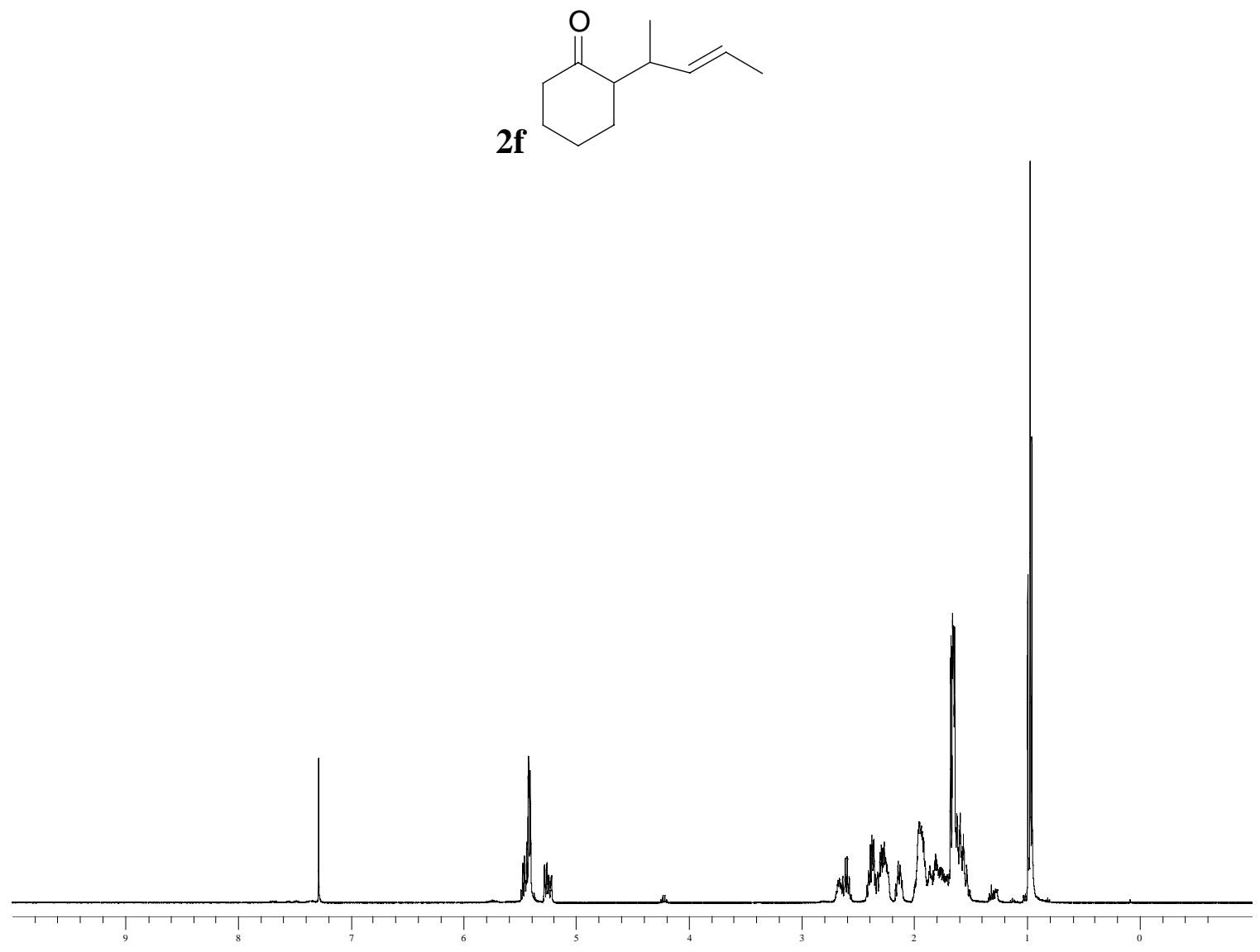




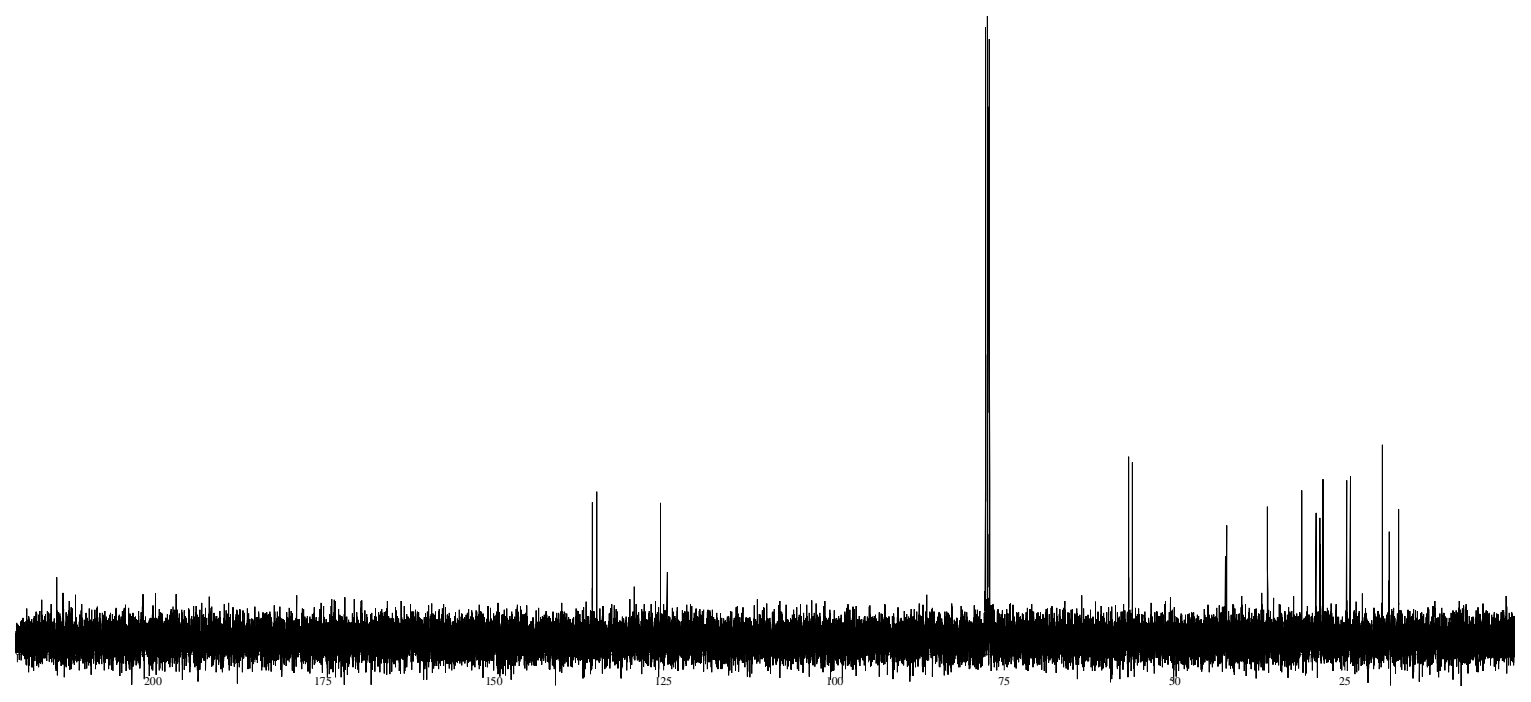

2f clear oil: ${ }^{1} \mathrm{H}$ NMR (400 MHz, $\mathrm{CDCl}_{3}$ ) Major Diastereomer: $\delta 5.37$ (overlapping multiplet, 1H: $\left.=\mathrm{CH}-\mathrm{CH}_{3}\right), 5.19(\mathrm{ddd}, \mathrm{J}=2 \mathrm{~Hz}, 8 \mathrm{~Hz}, 15 \mathrm{~Hz}, 1 \mathrm{H}:=\mathrm{CH}-\mathrm{CH}), 2.57$ (app. hex, $J=7 \mathrm{~Hz}, 1 \mathrm{H}$ : $\mathrm{CH}-\mathrm{CH}_{3}$ ), 2.27 (overlapping multiplet, cyclohexyl $\left.\mathrm{CH}_{2}\right), 2.12(\mathrm{~m}, 1 \mathrm{H}$ : $(\mathrm{C}=\mathrm{O}) \mathrm{CH}$ ), 1.93 (overlapping multiplet, cyclohexyl $\mathrm{CH}_{2}$ ), 1.92 (overlapping multiplet, cyclohexyl $\mathrm{CH}_{2}$ ), 1.65 (overlapping multiplet, $=\mathrm{CH}-\mathrm{CH}_{3}$ ), 1.59 (overlapping multiplet, cyclohexyl $\mathrm{CH}_{2}$ ), $0.97\left(\mathrm{~d}, J=6.6 \mathrm{~Hz}, 3 \mathrm{H}: \mathrm{CH}-\mathrm{CH}_{3}\right)$. Minor Diastereomer: $\delta 5.39$ (overlapping multiplet, $=\mathrm{CH}-\mathrm{CH}), 5.37$ (overlapping multiplet, $\left.=\mathrm{CH}-\mathrm{CH}_{3}\right), 2.65(\mathrm{~m}, 1 \mathrm{H}$ : $\left.\mathrm{CH}-\mathrm{CH}_{3}\right), 2.37$ (overlapping multiplet, cyclohexyl $\left.\mathrm{CH}_{2}\right), 2.23(\mathrm{~m}, 1 \mathrm{H}:(\mathrm{C}=\mathrm{O}) \mathrm{CH}), 1.84$ (overlapping multiplet, cyclohexyl $\mathrm{CH}_{2}$ ), 1.74 (overlapping multiplet, cyclohexyl $\mathrm{CH}_{2}$ ), 1.65 (overlapping multiplet, $=\mathrm{CH}-\mathrm{CH}_{3}$ ), 1.59 (overlapping multiplet, cyclohexyl $\mathrm{CH}_{2}$ ), 0.99 (d, $\left.J=6.6 \mathrm{~Hz}, 3 \mathrm{H}: \mathrm{CH}-\mathrm{CH}_{3}\right) .{ }^{13} \mathrm{C} \mathrm{NMR}\left(75 \mathrm{MHz}, \mathrm{CDCl}_{3}\right.$ ) Major Diastereomer: $\delta$ $214.03(\mathrm{C}=\mathrm{O}), 134.72(=\mathrm{CH}-\mathrm{CH}), 125.39\left(=\mathrm{CH}-\mathrm{CH}_{3}\right), 56.71((\mathrm{C}=\mathrm{O}) \mathrm{CH}), 42.32$ (2.27) (cyclohexyl $\left.\mathrm{CH}_{2}\right), 36.26\left(\mathrm{CH}-\mathrm{CH}_{3}\right), 31.32$ (1.93) (cyclohexyl $\left.\mathrm{CH}_{2}\right), 28.54$ (1.92) (cyclohexyl $\left.\mathrm{CH}_{2}\right), 24.20$ (1.59) (cyclohexyl $\left.\mathrm{CH}_{2}\right), 19.47\left(\mathrm{CH}-\mathrm{CH}_{3}\right), 18.36\left(=\mathrm{CH}-\mathrm{CH}_{3}\right)$. Minor Diastereomer: $\delta 213.12(\mathrm{C}=\mathrm{O}), 135.35(=\mathrm{CH}-\mathrm{CH}), 124.48\left(=\mathrm{CH}-\mathrm{CH}_{3}\right), 56.16$ $((\mathrm{C}=\mathrm{O}) \mathrm{CH}), 42.51$ (2.37) (cyclohexyl $\left.\mathrm{CH}_{2}\right), 35.47\left(\mathrm{CH}_{-} \mathrm{CH}_{3}\right), 29.21$ (1.59) (cyclohexyl $\mathrm{CH}_{2}$ ), 28.08 (1.74) (cyclohexyl $\left.\mathrm{CH}_{2}\right), 24.69$ (1.84) (cyclohexyl $\left.\mathrm{CH}_{2}\right), 18.39\left(=\mathrm{CH}-\mathrm{CH}_{3}\right)$, $17.00\left(\mathrm{CH}-\mathrm{CH}_{3}\right)$. IR $\left(\mathrm{CDCl}_{3}\right): v_{\max } 1700,1449$. HRMS calcd for $\mathrm{C}_{11} \mathrm{H}_{19} \mathrm{O}[\mathrm{M}+\mathrm{H}]$ 167.1436, found 167.1431. GC (Chiraldex B-TA : Hold $50^{\circ} \mathrm{C}$ for $5 \mathrm{~min}$., ramp $1^{\circ} \mathrm{C} / \mathrm{min}$ to $115^{\circ} \mathrm{C}$ ) Major Diastereomer: $t_{r}=67.3,69.8 \min$. Minor Diastereomer: $t_{r}=68.5$, $68.8 \mathrm{~min}$.

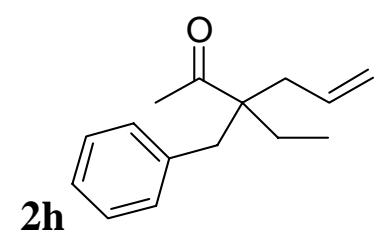



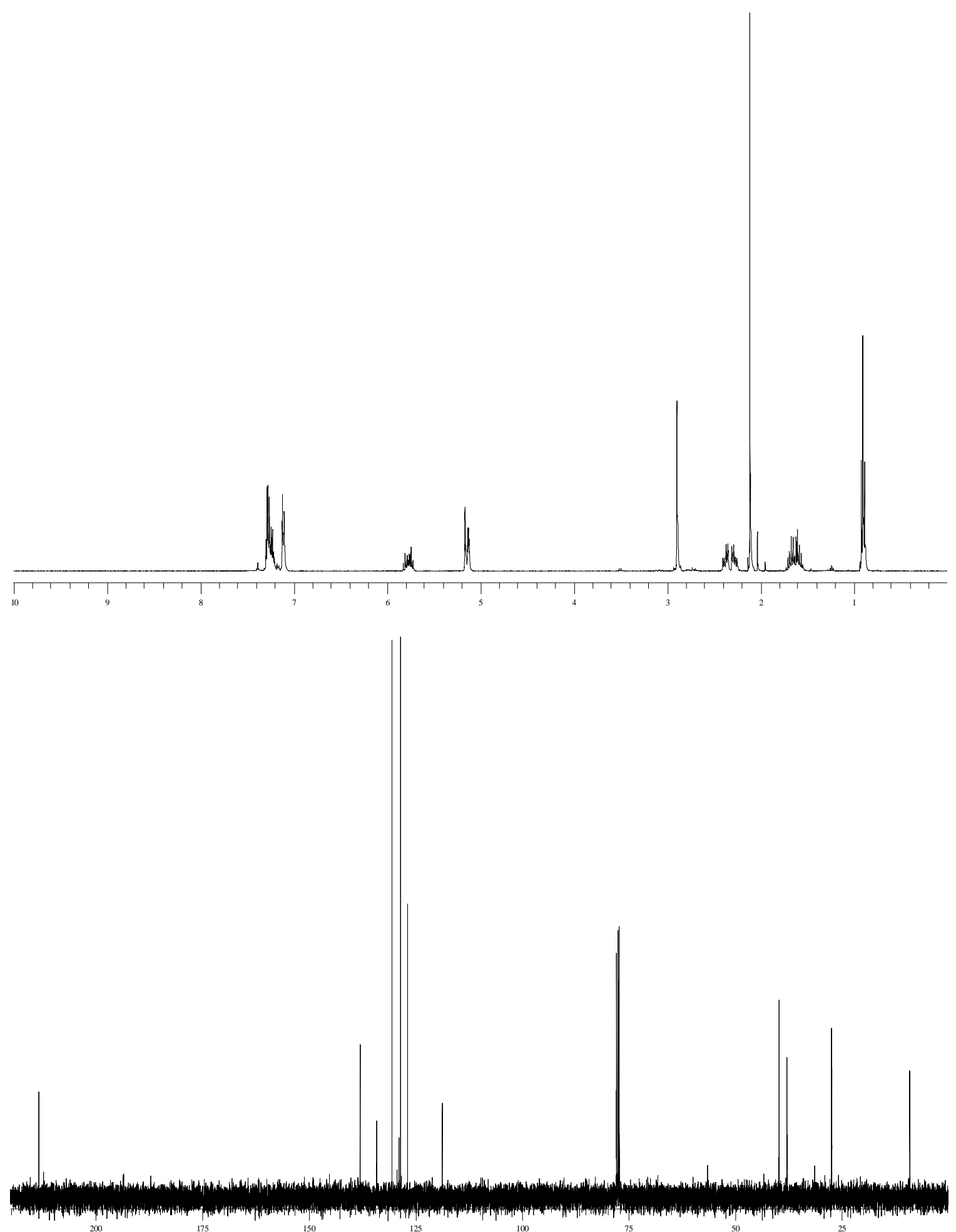

2h clear oil: ${ }^{1} \mathrm{H}$ NMR $\left(400 \mathrm{MHz}, \mathrm{CDCl}_{3}\right) \delta 7.26(\mathrm{~m}, 3 \mathrm{H}$ : arom $\mathrm{H}), 7.11(\mathrm{~d}, J=8 \mathrm{~Hz}, 2 \mathrm{H}$ : $\operatorname{arom~} \mathrm{H}), 5.77(\mathrm{~m}, 1 \mathrm{H}:=\mathrm{CH}), 5.14\left(\mathrm{~d}, J=12 \mathrm{~Hz}, 1 \mathrm{H}:=\mathrm{CH}(H)_{\text {cis }}\right), 5.14(\mathrm{~d}, J=17 \mathrm{~Hz}, 1 \mathrm{H}$ : $\left.=\mathrm{CH}(H)_{\text {trans }}\right), 2.89\left(\mathrm{~s}, 2 \mathrm{H}: \mathrm{CH}_{2} \mathrm{Ph}\right), 2.38\left(\mathrm{dd}, \mathrm{J}=7 \mathrm{~Hz}, 15 \mathrm{~Hz}, 1 \mathrm{H}\right.$ : diastereotopic $\mathrm{CH}_{2^{-}}$ $\mathrm{CH}=), 2.28\left(\mathrm{dd}, J=7 \mathrm{~Hz}, 15 \mathrm{~Hz}, 1 \mathrm{H}\right.$ : diastereotopic $\left.\mathrm{CH}_{2}-\mathrm{CH}=\right), 2.11\left(\mathrm{~s}, 3 \mathrm{H}:(\mathrm{C}=\mathrm{O})-\mathrm{CH}_{3}\right)$, 1.64 (m, 2H: $\left.\mathrm{CH}_{2}-\mathrm{CH}_{3}\right), 0.90\left(\mathrm{t}, J=7 \mathrm{~Hz}, 3 \mathrm{H}: \mathrm{CH}_{2}-\mathrm{CH}_{3}\right) .{ }^{13} \mathrm{C} \mathrm{NMR}\left(75 \mathrm{MHz}, \mathrm{CDCl}_{3}\right) \delta$ $213.40(\mathrm{C}=\mathrm{O}), 138.01$ (arom. C), $134.10(=\mathrm{CH}), 130.45$ (arom. $\mathrm{CH}), 128.58$ (arom. $\mathrm{CH})$, 
126.82 (arom. CH), $118.72\left(=\mathrm{CH}_{2}\right), 56.42(\mathrm{C}), 39.67\left(\mathrm{CH}_{2}-\mathrm{Ph}\right), 37.81\left(\mathrm{CH}_{2}=\mathrm{CH}-\mathrm{CH}_{2}\right)$, $27.31\left((\mathrm{C}=\mathrm{O})-\mathrm{CH}_{3}\right), 27.26\left(\mathrm{CH}_{2}-\mathrm{CH}_{3}\right), 8.96\left(\mathrm{CH}_{2}-\mathrm{CH}_{3}\right) . \mathrm{IR}\left(\mathrm{CDCl}_{3}\right): v_{\max } 1699,1454$, 1261, 1269. HRMS calcd for $\mathrm{C}_{15} \mathrm{H}_{21} \mathrm{O}[\mathrm{M}+\mathrm{H}] 2$ 217.1592, found 217.1602.

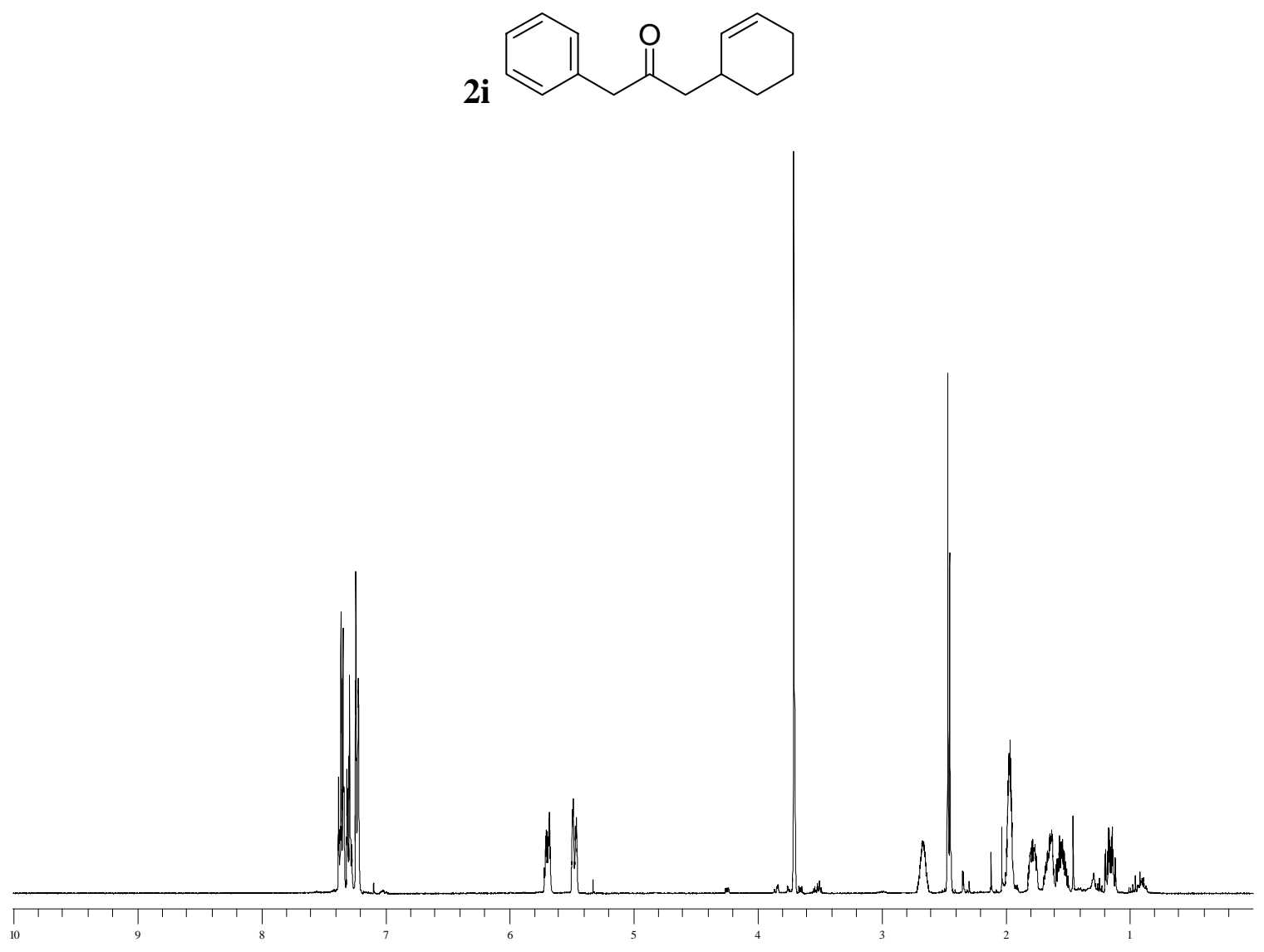




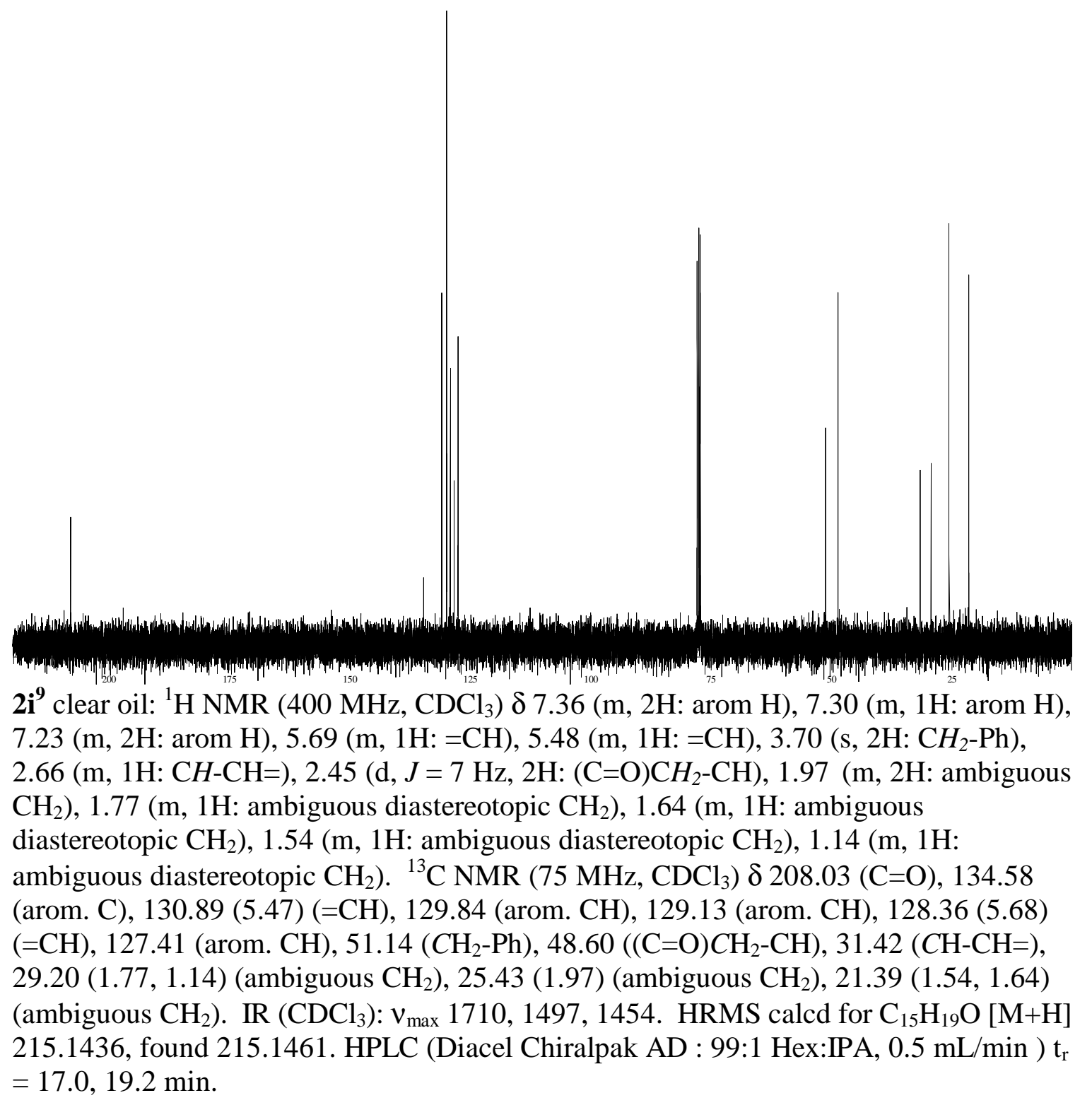<smiles>CC(C)C(=O)CC1CCCCC1</smiles>

\footnotetext{
${ }^{9}$ House, H.; Fischer, W.; Gall, M.; McLaughlin, T.; Peet, N. J. Org. Chem. 1971, 36, 3429-37.
} 

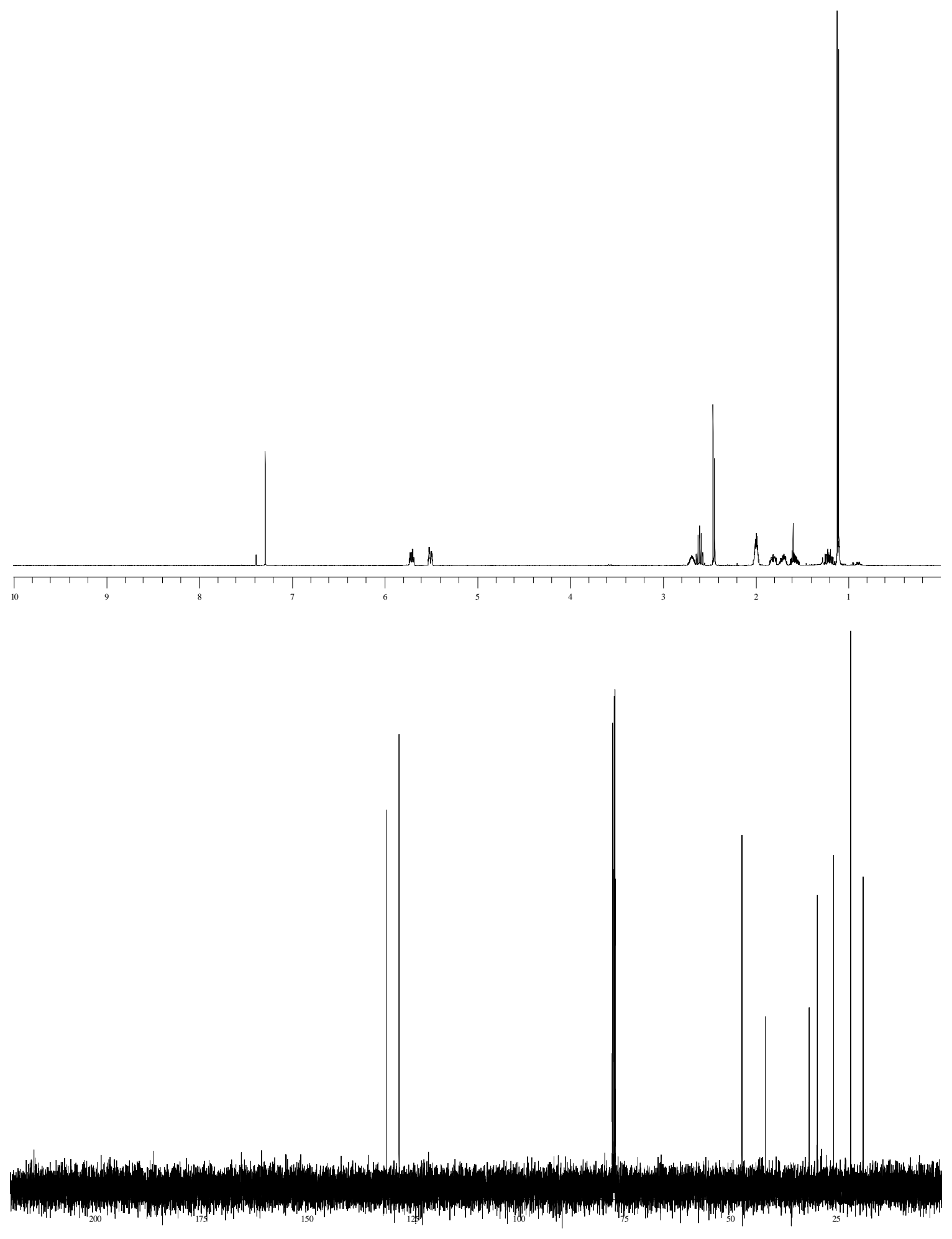

2j clear oil: ${ }^{1} \mathrm{H}$ NMR $\left(400 \mathrm{MHz}, \mathrm{CDCl}_{3}\right) \delta 5.71(\mathrm{~m}, 1 \mathrm{H}:=\mathrm{CH}), 5.50(\mathrm{~m}, 1 \mathrm{H}:=\mathrm{CH}), 2.68$ $(\mathrm{m}, 1 \mathrm{H}:=\mathrm{CH}-\mathrm{CH}), 2.61$ (app. pen, $\left.J=7 \mathrm{~Hz}, 1 \mathrm{H}:\left(\mathrm{CH}_{3}\right)_{2}-\mathrm{CH}\right), 2.45(\mathrm{~d}, J=7 \mathrm{~Hz}, 2 \mathrm{H}$ : $\left.(\mathrm{C}=\mathrm{O}) \mathrm{CH}_{2}\right), 1.99\left(\mathrm{~m}, 2 \mathrm{H}\right.$ : ambiguous $\left.\mathrm{CH}_{2}\right), 1.81(\mathrm{~m}, 1 \mathrm{H}$ : ambiguous diastereotopic $\left.\mathrm{CH}_{2}\right), 1.69$ (m, 1H: ambiguous diastereotopic $\left.\mathrm{CH}_{2}\right), 1.58$ (m, $1 \mathrm{H}$ : ambiguous 
diastereotopic $\left.\mathrm{CH}_{2}\right), 1.20$ (m, 1H: ambiguous diastereotopic $\left.\mathrm{CH}_{2}\right), 1.11(\mathrm{~d}, J=7 \mathrm{~Hz}, 6 \mathrm{H}$ : $\left.\left(\mathrm{CH}_{3}\right)_{2}\right) .{ }^{13} \mathrm{C} \mathrm{NMR}\left(75 \mathrm{MHz}, \mathrm{CDCl}_{3}\right) \delta 214.49(\mathrm{C}=\mathrm{O}), 131.23(5.50)(=\mathrm{CH}), 128.17$ (5.71) $(=\mathrm{CH}), 47.09\left((\mathrm{C}=\mathrm{O}) \mathrm{CH}_{2}\right), 41.61\left(\left(\mathrm{CH}_{3}\right)_{2}-\mathrm{CH}\right), 31.37(\mathrm{CH}-\mathrm{CH}=), 29.39$ (1.81, 1.20) (ambiguous $\mathrm{CH}_{2}$ ), 25.49 (1.99) (ambiguous $\mathrm{CH}_{2}$ ), $21.48(1.69,1.58)$ (ambiguous $\left.\mathrm{CH}_{2}\right), 18.52\left(\left(\mathrm{CH}_{3}\right)_{2}\right)$. IR $\left(\mathrm{CDCl}_{3}\right): v_{\max } 1707,1265,1467$. HPLC (Diacel Chiralpak AD : 99.5:1 Hex:IPA, $0.5 \mathrm{~mL} / \mathrm{min}) \mathrm{t}_{\mathrm{r}}=9.7,10.5 \mathrm{~min}$.

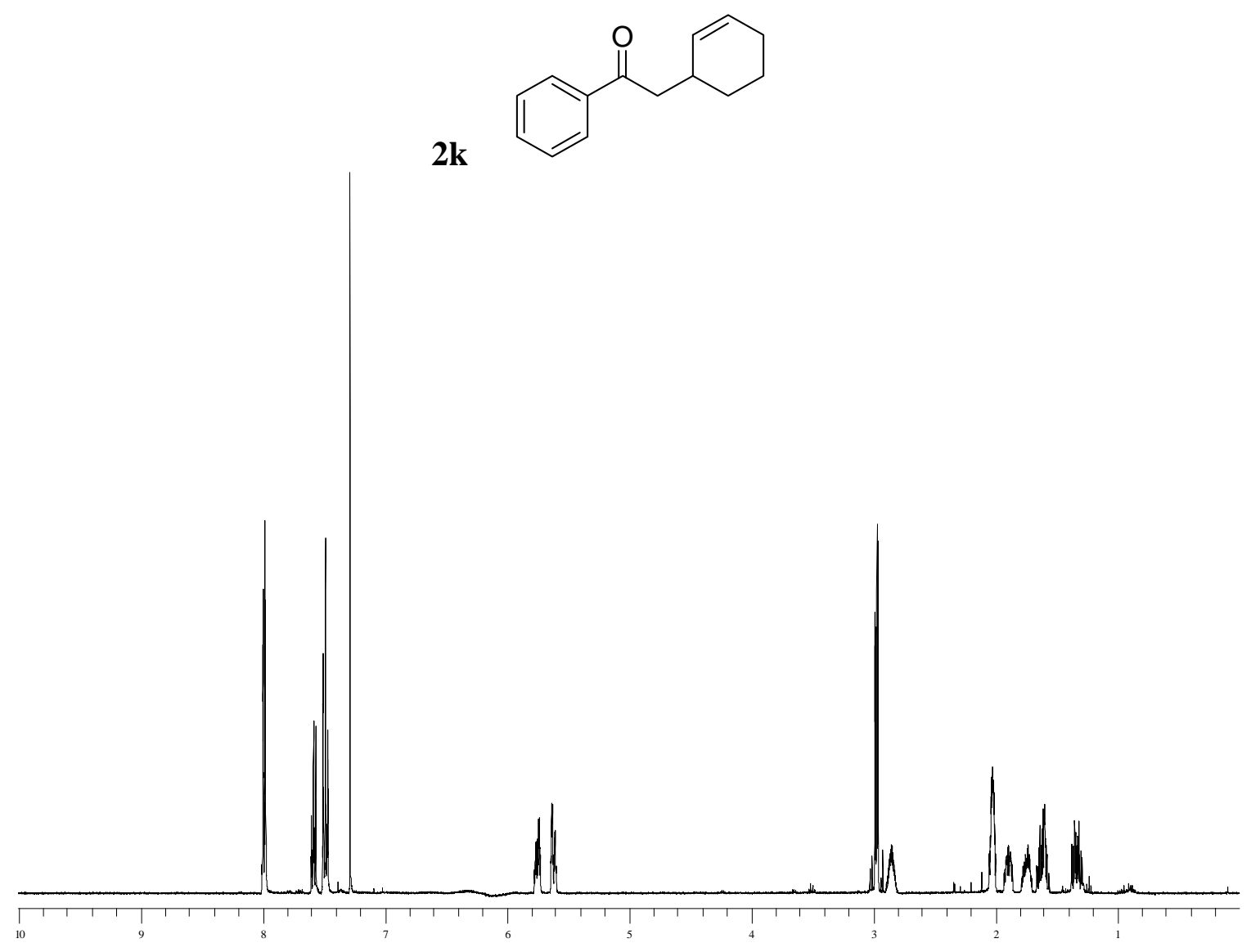




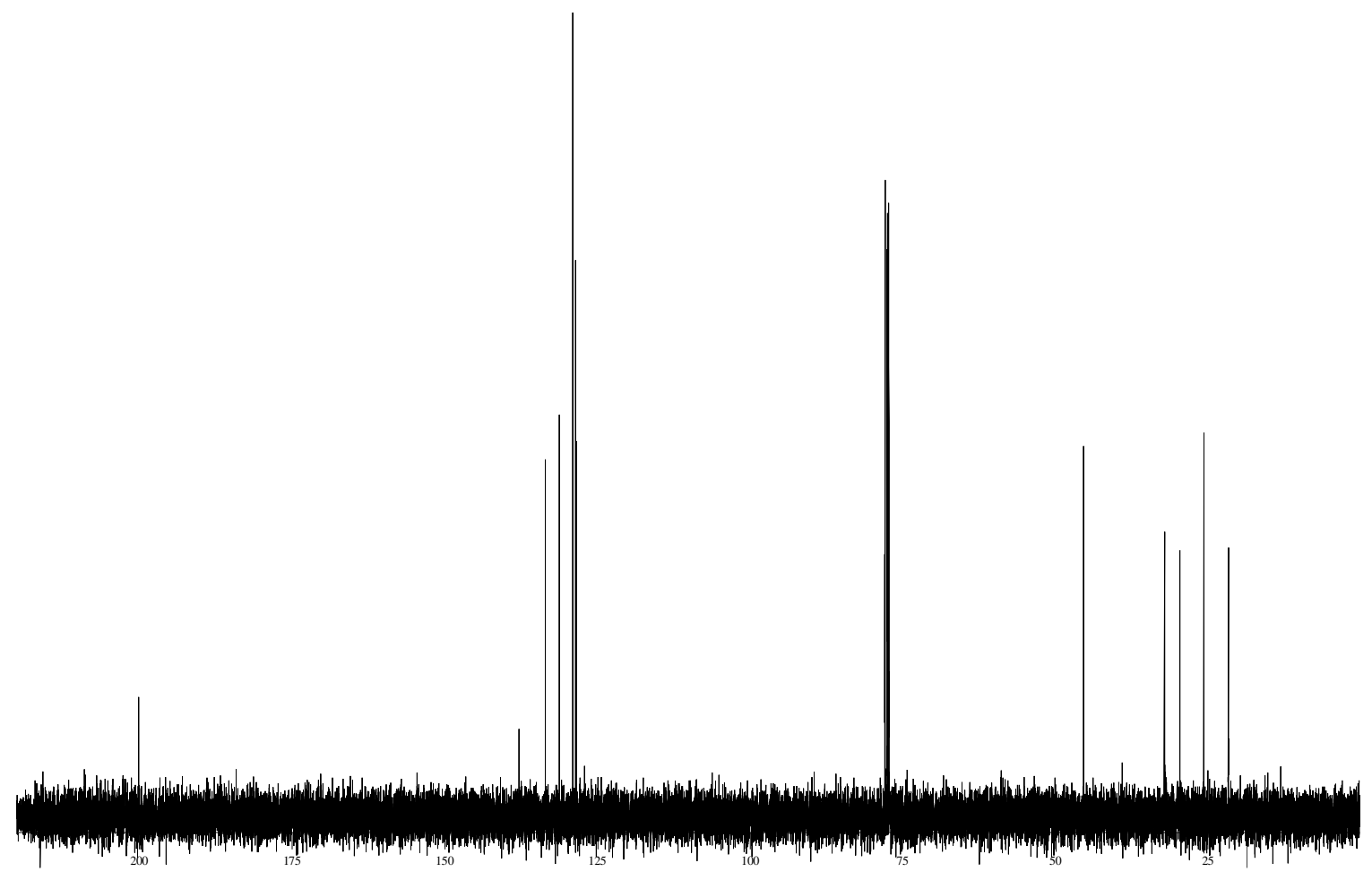

2k ${ }^{10}$ clear oil: ${ }^{1} \mathrm{H}$ NMR (400 MHz, $\left.\mathrm{CDCl}_{3}\right) \delta 7.99(\mathrm{~m}, 2 \mathrm{H}$ : arom $\mathrm{H}), 7.59$ (m, 1H: arom $\mathrm{H}), 7.49$ (app. t, J = 7 Hz, 2H: arom H), $5.75(\mathrm{~m}, 1 \mathrm{H}:=\mathrm{CH}), 5.61(\mathrm{~m}, 1 \mathrm{H}:=\mathrm{CH}), 2.98(\mathrm{~d}$, $J=7 \mathrm{~Hz}, 1 \mathrm{H}$ : diastereotopic $\left.(\mathrm{C}=\mathrm{O}) \mathrm{CH}_{2}\right), 2.97(\mathrm{~d}, J=7 \mathrm{~Hz}, 1 \mathrm{H}$ : diastereotopic

$\left.(\mathrm{C}=\mathrm{O}) \mathrm{CH}_{2}\right), 2.85(\mathrm{~m}, 1 \mathrm{H}$ : $=\mathrm{CH}-\mathrm{CH}), 2.02\left(\mathrm{~m}, 2 \mathrm{H}\right.$ : ambiguous $\left.\mathrm{CH}_{2}\right), 1.89(\mathrm{~m}, 1 \mathrm{H}$ : ambiguous diastereotopic $\left.\mathrm{CH}_{2}\right), 1.73\left(\mathrm{~m}, 1 \mathrm{H}\right.$ : ambiguous diastereotopic $\left.\mathrm{CH}_{2}\right), 1.61(\mathrm{~m}$, 1H: ambiguous diastereotopic $\left.\mathrm{CH}_{2}\right), 1.34$ (m, $1 \mathrm{H}$ : ambiguous diastereotopic $\left.\mathrm{CH}_{2}\right) .{ }^{13} \mathrm{C}$ NMR (75 MHz, $\left.\mathrm{CDCl}_{3}\right) \delta 200.08(\mathrm{C}=\mathrm{O}), 137.72$ (arom. C), 133.37 (arom. CH), 131.17 (5.61) $(=\mathrm{CH}), 128.98$ (arom. CH), 128.52 (arom. $\mathrm{CH}), 128.37$ (5.75) $(=\mathrm{CH}), 45.23$ $\left((\mathrm{C}=\mathrm{O}) \mathrm{CH}_{2}\right), 32.02(\mathrm{CH}-\mathrm{CH}=), 29.49(1.89,1.34)$ (ambiguous $\left.\mathrm{CH}_{2}\right), 25.53$ (2.02) (ambiguous $\left.\mathrm{CH}_{2}\right), 21.50(1.61,1.73)$ (ambiguous $\left.\mathrm{CH}_{2}\right)$. IR $\left(\mathrm{CDCl}_{3}\right): v_{\max } 1684,1448$, 1261. HRMS calcd for $\mathrm{C}_{14} \mathrm{H}_{17} \mathrm{O}[\mathrm{M}+\mathrm{H}]$ 201.1279, found 201.1278. HPLC (Diacel Chiralpak AD : 99:1 Hex:IPA, 0.5 mL/min ) $\mathrm{t}_{\mathrm{r}}=15.3,16.0 \mathrm{~min}$.<smiles>O=C(C[Te])CC1C=CCC1</smiles>

\footnotetext{
${ }^{10}$ Fernandez-Mateos, A; Alonso, J.; Gonzalez, R. Tetrahedron. 1999, 55, 847-860.
} 

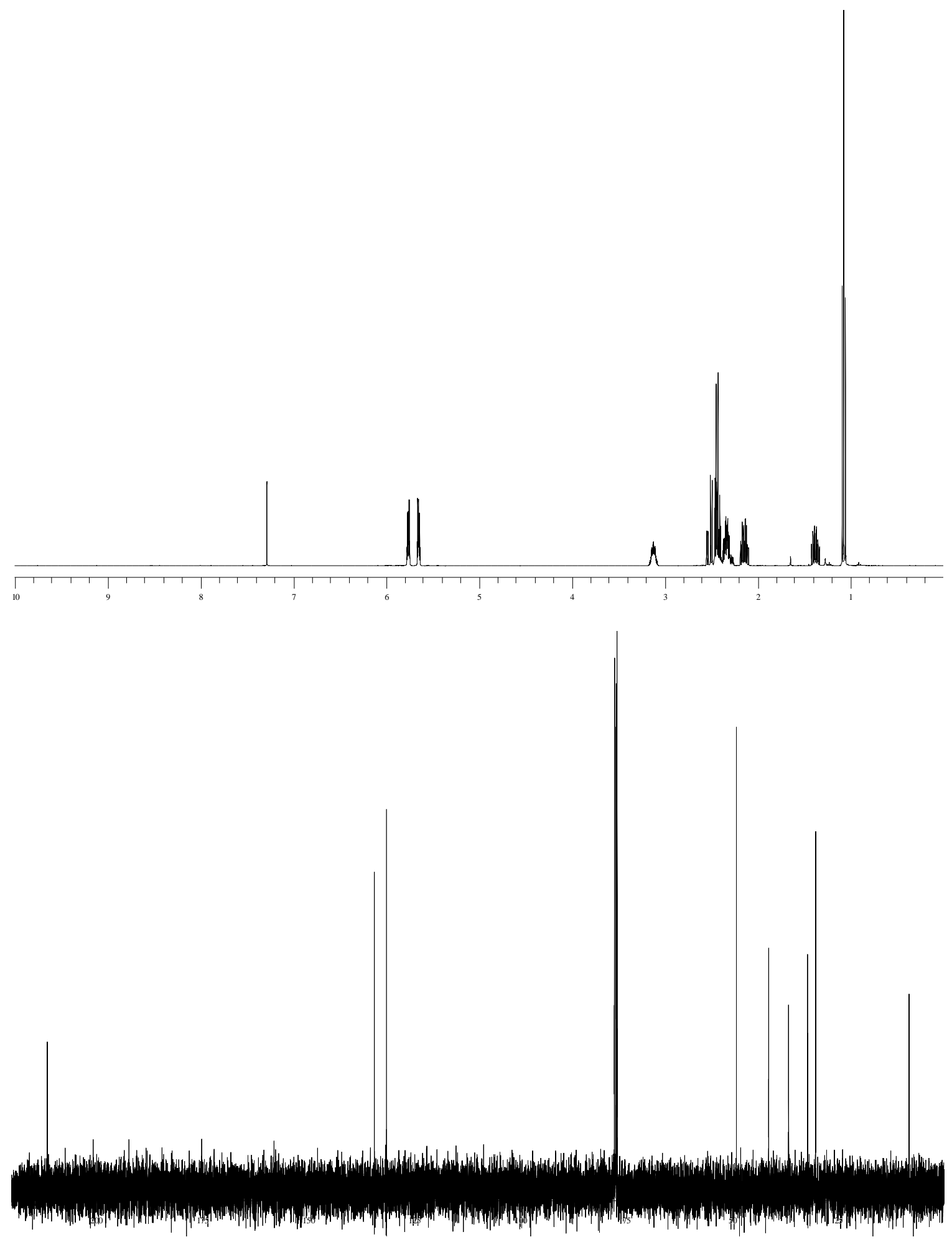

2l clear oil: ${ }^{1} \mathrm{H}$ NMR $\left(400 \mathrm{MHz}, \mathrm{CDCl}_{3}\right) \delta 5.76(\mathrm{~m}, 1 \mathrm{H}:=\mathrm{CH}), 5.65(\mathrm{~m}, 1 \mathrm{H}:=\mathrm{CH}), 3.12$ (m, 1H: $\mathrm{CH}-\mathrm{CH}=), 2.52\left(\mathrm{dd}, J=7 \mathrm{~Hz}, 16.5 \mathrm{~Hz}, 1 \mathrm{H}\right.$ : diastereotopic $\left.(\mathrm{C}=\mathrm{O}) \mathrm{CH}_{2}\right), 2.43$ (overlapping multiplet, $1 \mathrm{H}$ : diastereotopic $(\mathrm{C}=\mathrm{O}) \mathrm{CH}_{2}$ ), 2.43 (overlapping multiplet, $2 \mathrm{H}$ : $\left.\mathrm{CH}_{2}-\mathrm{CH}_{3}\right), 2.33\left(\mathrm{~m}, 2 \mathrm{H}\right.$ : ambiguous $\left.\mathrm{CH}_{2}\right), 2.14$ (m, 1H: ambiguous diastereotopic $\mathrm{CH}_{2}$ ), 
1.38 (m, 1H: ambiguous diastereotopic $\left.\mathrm{CH}_{2}\right), 1.07\left(\mathrm{t}, J=7.3 \mathrm{~Hz}, 3 \mathrm{H}: \mathrm{CH}_{3}\right) .{ }^{13} \mathrm{C} \mathrm{NMR}$ $\left(75 \mathrm{MHz}, \mathrm{CDCl}_{3}\right) \delta 211.63(\mathrm{C}=\mathrm{O}), 134.48(5.65)(=\mathrm{CH}), 131.56(5.76)(=\mathrm{CH}), 48.99$ $\left((\mathrm{C}=\mathrm{O}) \mathrm{CH}_{2}\right), 41.43(\mathrm{CH}-\mathrm{CH}=), 36.70\left(\mathrm{CH}_{2}-\mathrm{CH}_{3}\right), 32.22(2.33)$ (ambiguous $\left.\mathrm{CH}_{2}\right), 30.28$ (1.38) (ambiguous $\left.\mathrm{CH}_{2}\right), 8.21\left(\mathrm{CH}_{3}\right)$. IR $\left(\mathrm{CDCl}_{3}\right): v_{\max } 1710,1460,1115$. HRMS calcd for $\mathrm{C}_{9} \mathrm{H}_{14} \mathrm{O}[\mathrm{M}+\mathrm{H}]$ 166.1358, found 166.0780. GC (Chiraldex B-TA : Hold $50^{\circ} \mathrm{C}$ for 5 min., ramp $0.5^{\circ} \mathrm{C} / \mathrm{min}$ to $\left.75^{\circ} \mathrm{C}\right) \mathrm{t}_{\mathrm{r}}=60.1,62.1 \mathrm{~min}$. 